May, 1993

IFUP-TH $17 / 93$

\title{
The Three-Loop Lattice Free Energy
}

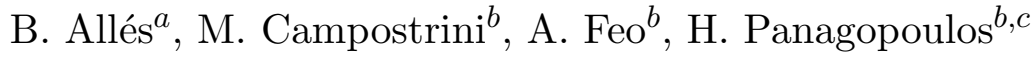 \\ a) Departamento de Física Teórica y del Cosmos, Universidad de Granada, Spain \\ b) I.N.F.N. and Dipartimento di Fisica dell'Università, Pisa, Italy \\ c) Dept. of Natural Sciences, Univ. of Cyprus, Nicosia, Cyprus
}

\begin{abstract}
We calculate the free energy of $\mathrm{SU}(N)$ gauge theories on the lattice, to three loops. Our result, combined with Monte Carlo data for the average plaquette, gives a more precise estimate of the gluonic condensate.
\end{abstract}


In a previous work [1] we described a scheme for carrying out perturbative calculations on the lattice, using a symbolic manipulation language. We also used this scheme to obtain the first three-loop result, related to the topological susceptibility.

In this paper, we present a three-loop calculation of the free energy in pure $\mathrm{SU}(N)$ gauge theories. Using this perturbative result together with Monte Carlo data for the average plaquette we arrive at a more precise determination of the (non- perturbative) gluon condensate

$$
G_{2}=-\frac{\beta(g)}{g} \frac{1}{4 \pi^{2} b_{0}} \sum_{a, \mu, \nu} F_{\mu \nu}^{a} F_{\mu \nu}^{a} .
$$

This condensate has been widely used in phenomenological studies of QCD, in particular in the context of the SVZ sum rules [2]. In addition, the feasibility of extracting $G_{2}$ from a lattice simulation is a challenging theoretical issue on its own right; the presence of mixing with lower dimensional operators $\left(F_{\mu \nu} F^{\mu \nu}\right.$ mixes with the unit operator) represents a priori a major stumbling block. It is important to see just to what accuracy this extraction can be pushed given the systematic error.

The gluonic condensate is related to Monte Carlo data through the expression

$$
\langle\Pi\rangle_{\mathrm{MC}}=Z(\beta)\left(-\frac{\pi^{2}}{12}\right) G_{2} a^{4}+\langle\Pi\rangle_{\mathrm{pert}},
$$

where

$$
\beta=\frac{2 N}{g^{2}}
$$

and $\Pi$ is the plaquette operator

$$
\Pi \equiv \Pi_{x, \mu \nu}=\operatorname{tr}\left(U_{x, x+\mu} U_{x+\mu, x+\mu+\nu} U_{x+\mu+\nu, x+\nu} U_{x+\nu, x}\right) .
$$

The quantities $Z(\beta)$ and $\langle\Pi\rangle_{\text {pert }}$ can be calculated perturbatively; while $Z(\beta)$ for the plaquette operator is very close to 1 and can be left out to a rather good approximation, $\langle\Pi\rangle_{\text {pert }}$ is far from negligible, and it is important to determine it with the best possible precision.

We write:

$$
\langle\Pi\rangle_{\text {pert }}=N+\frac{c_{1}}{\beta}+\frac{c_{2}}{\beta^{2}}+\frac{c_{3}}{\beta^{3}}+\cdots,
$$

where $c_{1}=-N\left(N^{2}-1\right) / 4$ and $c_{2}$ was calculated in Ref. 3 .

We have carried out the calculation of the three-loop coefficient $c_{3}$. This a priori entails computing all vacuum diagrams with one insertion of the plaquette operator; there are 63 such distinct diagrams. The relationship between $\Pi$ and the Wilson action

$$
S=\sum_{x, \mu \nu}\left(N-\Pi_{x, \mu \nu}\right)
$$

allows a simplification of this task, by relating $\langle\Pi\rangle_{\text {pert }}$ to the partition function $Z=\int[d U] \exp (-\beta / 2 N S)$. We have:

$$
\begin{aligned}
\langle\Pi\rangle_{\text {pert }} & =\frac{1}{Z} \int[d U] e^{-\frac{\beta}{2 N} S} \Pi_{x, \mu \nu} \\
& =N-\frac{1}{12 V} \frac{1}{Z} \int[d U] e^{-\frac{\beta}{2 N} S} S=N+\frac{2 N}{12 V} \frac{\partial}{\partial \beta} \ln Z .
\end{aligned}
$$


Now, $\ln Z$ has the form

$$
\ln Z=V\left(-\frac{3\left(N^{2}-1\right)}{2} \ln \beta+\frac{d_{1}}{\beta}+\frac{d_{2}}{\beta^{2}}+\cdots\right),
$$

which implies that $c_{3}=-N / 2 d_{2}$. The calculation of $d_{2}$ involves computing all 3-loop connected vacuum diagrams with no operator insertions; there are 29 diagrams giving nonzero contributions altogether, shown in Fig. 2.

We have computed these diagrams using our algebraic package described in Ref. 1. The main building blocks of this package are:

i) Generating all relevant vertices.

ii) Performing contractions for each diagram and producing the corresponding integrand. Here, as well as in i), it is crucial to exploit all available symmetries, in order to render the resulting expressions more compact.

iii) Integrating over loop momenta, for infinite as well as finite lattices.

The contributions from each diagram in the Feynman gauge are given for reference in Table I. The final result, given in Table II, is, of course, gauge independent. Incidentally, the CPU time necessary for the actual runs (once the development and testing stages are over) is $\sim 3$ hours on a Sun SPARC ELC to produce the integrand for some of the more complicated diagrams and $\sim 2$ hours for a highly optimized integration on lattices up to $16^{4}$. (A notable exception are diagrams with the topology of Fig. 21, known as "Mercedes-Benz emblem"; the integration of these diagrams requires $\sim 20$ hours already for an $8^{4}$ lattice.)

A preliminary presentation of the results reported here was given in Ref. 4. Note however a discrepancy due to an error in the preliminary version.

Using numerical results for $L^{4}$ lattices $(L=2, \ldots, 16)$ we can extrapolate out to $L=\infty$ with a functional form:

$$
r(L)=r_{0}+\frac{r_{i}}{L^{i}}\left(+\frac{r_{j}}{L^{j}}\right) \quad i, j=2,3,4
$$

depending on the diagram, and compare with the infinite lattice results $r(\infty)$. (Infinite lattice results are obtained with the Schwinger representation technique, explained in Ref. 1.) The discrepancy is typically a fraction of a per mille, so that $r(L)$ can be used also for lattices with $L>16$. The actual analytic form of $r(L)$, for $L \rightarrow \infty$, could also involve terms proportional to $(\ln L)^{n} / L^{m}[5]$; however the introduction of these terms does not alter our extrapolations. It should also be mentioned here that, while lattice integrals are ultraviolet convergent, individual diagrams may exhibit infrared divergences, which cancel only when they are summed up. For the case at hand, the diagrams in Fig. 2o, containing a double insertion of the one-loop renormalized gluon propagator (Fig. 1), must be evaluated simultaneously, in order to guarantee infrared convergence.

Having obtained the value of $c_{3}$ we can now reanalyze Monte Carlo data for $\mathrm{SU}(2)$ [6], 17] and $\mathrm{SU}(3)$ [8], using Eq. (2), to extract the gluon condensate. We perform two series of $\chi^{2}$ fits, one in which $c_{3}$ is a parameter to be fitted, the other using the calculated value of $c_{3}$ and fitting instead $c_{4}$. The results are listed in Table III; in all cases we have $\chi^{2} /$ d.o.f. $\simeq 1$. It is reassuring to see that, despite variations in the fitted values of the parameters $c_{i}$, the condensate exhibits a very stable behavior.

To conclude, our algebraic scheme has made possible this 3-loop calculation; done by hand, such a calculation would be extremely cumbersome, if not downright impossible. At 
the present stage of development of this scheme, one can compute vacuum expectation values of any operator to an arbitrary number of loops (modulo computer limitations). We are currently extending the scheme to include matrix elements with external fields; the main problem there is extracting the analytic dependence on external momenta, which can be a rather delicate issue beyond one loop. We will report on this subject in a future publication.

Acknowledgments. It is a pleasure to thank Adriano Di Giacomo for many useful conversations. We acknowledge financial support from MURST (Italian Ministry of the University and of Scientific and Technological Research) and from the Spanish-Italian "Integrated Action" (contract A17). B.A. also acknowledges a Spanish CICYT contract.

\section{References}

1. B. Allés, M. Campostrini, A. Feo, and H. Panagopoulos, Lattice Perturbation Theory by Computer Algebra: A Three-Loop Result for the Topological Susceptibility, Pisa preprint IFUP-TH 31/92.

2. M. A. Shifman, A. I. Vainshtein, and V. I. Zakharov, Nucl. Phys. B147 (1979) 385, 448, 519.

3. A. Di Giacomo and G. C. Rossi, Phys. Lett. B100 (1981) 481.

4. B. Allés, M. Campostrini, A. Feo, and H. Panagopoulos, Proc. of the Lattice Gauge Theory '92 Conference, Nucl. Phys. B (Proc. Suppl.) 30 (1993) 243.

5. M. Lüscher and P. Weisz, Nucl. Phys. B266 (1986) 309.

6. M. Campostrini, G. Curci, A. Di Giacomo, and G. Paffuti, Zeitschr. Phys. C32 (1986) 377.

7. A. Di Giacomo, H. Panagopoulos, and E. Vicari, Nucl. Phys. B338 (1990) 294.

8. M. Campostrini, A. Di Giacomo, and Y. Gündüc̣, Phys. Lett. 225B (1989) 393. 


\section{Table I}

The contribution to $c_{3}$ of individual diagrams, shown in Fig. 2, in the Feynman gauge. We use an $L^{4}$ lattice and gauge group $\mathrm{SU}(N)$. Each entry must be multiplied by $N^{5}\left(N^{2}-1\right) 10^{-4}$.

\begin{tabular}{|c|c|c|c|c|}
\hline Fig. & $L=8$ & $L=12$ & $L=16$ & $L=\infty$ \\
\hline $\mathrm{a}$ & $41.874-93.004 / N^{2}$ & $41.833-92.971 / N^{2}$ & $41.811-92.946 / N^{2}$ & $41.775-92.902 / N^{2}$ \\
& $+52.045 / N^{4}$ & $+52.076 / N^{4}$ & $+52.081 / N^{4}$ & $+52.083 / N^{4}$ \\
$\mathrm{~b}$ & 1.0803 & 1.0974 & 1.1035 & 1.1113 \\
$\mathrm{c}$ & $38.582-43.395 / N^{2}$ & $40.013-44.714 / N^{2}$ & $40.528-45.184 / N^{2}$ & $41.200-45.795 / N^{2}$ \\
$\mathrm{~d}$ & 1.0806 & 1.0975 & 1.1035 & 1.1113 \\
$\mathrm{e}$ & 1.0803 & 1.0974 & 1.1035 & 1.1113 \\
$\mathrm{f}$ & 0.22019 & 0.23398 & 0.23894 & 0.24543 \\
$\mathrm{~g}$ & 0.018161 & 0.021014 & 0.022139 & 0.023730 \\
$\mathrm{~h}$ & -10.464 & -10.696 & -10.781 & -10.892 \\
& $+\left(1 / N^{2}-3 / N^{4}\right) 26.993$ & $+\left(1 / N^{2}-3 / N^{4}\right) 27.013$ & $+\left(1 / N^{2}-3 / N^{4}\right) 27.017$ & $+\left(1 / N^{2}-3 / N^{4}\right) 27.019$ \\
$\mathrm{i}$ & 0.41123 & 0.41825 & 0.42074 & 0.42394 \\
$\mathrm{j}$ & -1.7753 & -1.7876 & -1.7880 & -1.7839 \\
$\mathrm{k}$ & 0.084525 & 0.089997 & 0.091975 & 0.094566 \\
$\mathrm{l}$ & -0.50731 & -0.56679 & -0.58988 & -0.62246 \\
$\mathrm{~m}$ & 0.023925 & 0.027060 & 0.028245 & 0.029881 \\
$\mathrm{n}$ & 0.036795 & 0.037688 & 0.038022 & 0.038479 \\
$\mathrm{o}$ & $-135.184+290.471 / N^{2}$ & $-136.433+291.877 / N^{2}$ & $-136.861+292.342 / N^{2}$ & $-137.404+292.919 / N^{2}$ \\
& $-156.136 / N^{4}$ & $-156.227 / N^{4}$ & $-156.243 / N^{4}$ & $-156.25 / N^{4}$ \\
\hline Total & $-63.438+181.064 / N^{2}$ & $-63.517+181.204 / N^{2}$ & $-63.532+181.229 / N^{2}$ & $-63.537+181.240 / N^{2}$ \\
& $-185.068 / N^{4}$ & $-185.191 / N^{4}$ & $-185.211 / N^{4}$ & $-185.223 / N^{4}$ \\
\hline
\end{tabular}

Table II

The calculated, gauge independent value of $c_{3}$ in $\mathrm{SU}(2)$ and $\mathrm{SU}(3)$.

\begin{tabular}{|c|c|c|c|c|}
\hline$c_{3}$ & $L=8$ & $L=12$ & $L=16$ & $L=\infty$ \\
\hline$N=2$ & -0.28549 & -0.28599 & -0.28608 & -0.28611 \\
$N=3$ & -8.8655 & -8.8781 & -8.8805 & -8.8814 \\
\hline
\end{tabular}

Table III

We list the values of $\pi^{2} / 12 N G_{2} / \Lambda_{\mathrm{QCD}}^{4}$ (for gauge groups $\mathrm{SU}(2)(\beta=2.45)$ and $\mathrm{SU}(3)$ $(\beta=6.0))$, as obtained from Eq. (2) and Monte Carlo data through a series of fits, in which: a) $c_{3}$ was an additional parameter to be fitted, or: b) the exact value of $c_{3}$ was taken from our calculation, fitting instead $c_{4}$.

\begin{tabular}{|c|c|c|}
\hline$\pi^{2} / 12 N G_{2} / \Lambda_{\mathrm{QCD}}^{4}$ & $\mathrm{SU}(2)$ & $\mathrm{SU}(3)$ \\
\hline $\mathrm{a}$ & $1.09(09) 10^{7}$ & $2.79(33) 10^{8}$ \\
$\mathrm{~b}$ & $1.06(10) 10^{7}$ & $2.39(61) 10^{8}$ \\
\hline
\end{tabular}




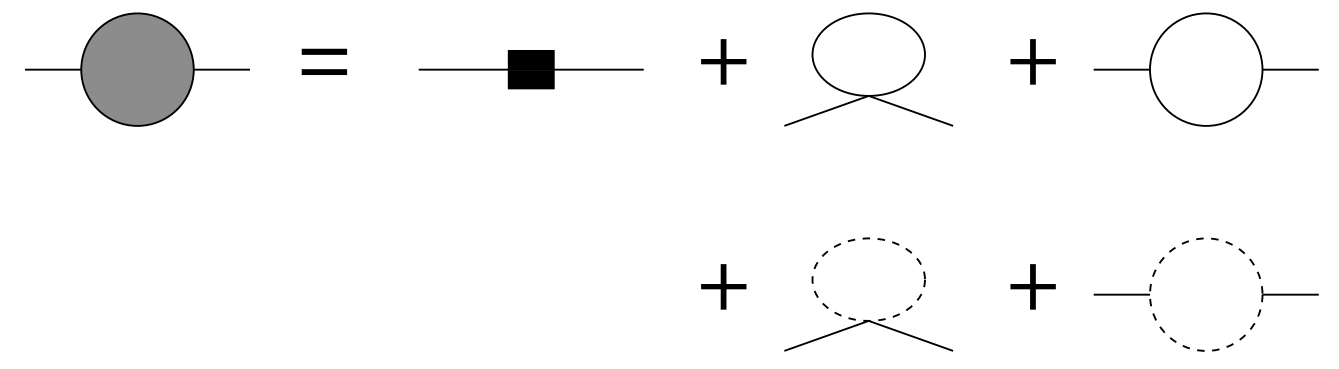

Fig. 1 The one-loop gluon propagator. A square stands for the integration measure.

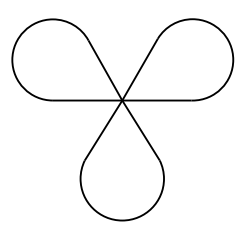

a)

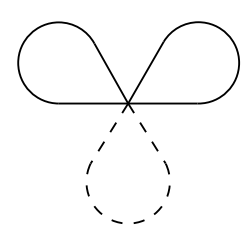

b)

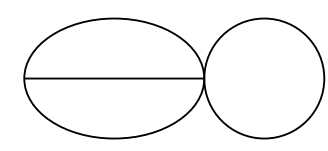

c)

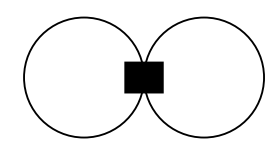

d)

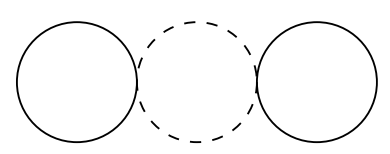

e)

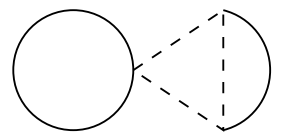

f)

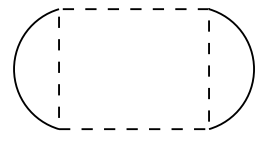

g)

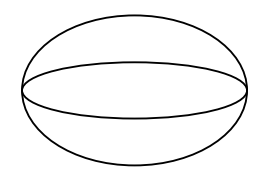

h)

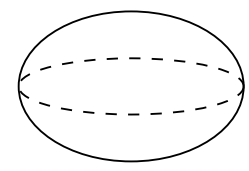

i)

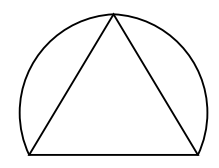

j)

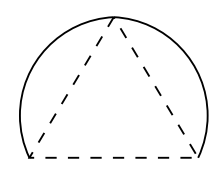

k)

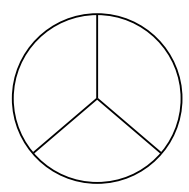

l)

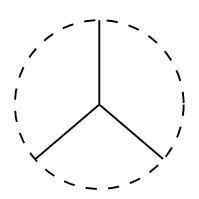

m)

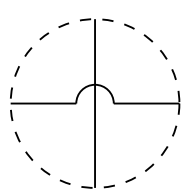

n)

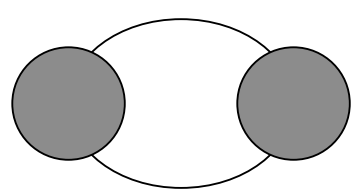

o)

Fig. 2 Diagrams contributing to $c_{3}$. 